\title{
Technical note: Assessment of neutrophil endocytosis and proteolytic degradation and its relationship with phagocytosis and oxidative burst in dairy cows
}

\author{
O. Bogado Pascottini, ${ }^{1 *}$ M. Baquero, ${ }^{2}$ R. Couto Serrenho, ${ }^{1}$ S. J. Van Schyndel, ${ }^{1}$ D. Bienzle, ${ }^{2}$ and S. J. LeBlanc ${ }^{1}$ \\ ${ }^{1}$ Department of Population Medicine, University of Guelph, Guelph, ON, Canada N1G 2W1 \\ ${ }^{2}$ Department of Pathobiology, University of Guelph, Guelph, ON, Canada N1G 2W1
}

\section{ABSTRACT}

Conventional assays of polymorphonuclear cell (PMN, neutrophil) function such as oxidative burst (OB) and phagocytosis (PC) are widely used to evaluate innate immunity in the transition period of dairy cows. Oxidative burst is commonly evaluated by measuring PMN median fluorescence intensity (MFI) involving the release of reactive oxygen species after in vitro stimulation. Phagocytosis can be measured by engulfment of fluorescent beads by PMN. DQ-ovalbumin (DQ-OVA) is a molecule suitable for the assessment of intracellular proteolytic degradation, so it might be informative about an additional pathway of pathogen handling by PMN. In this study, we evaluated the use of the DQOVA assay for the assessment of PMN function and the relationships among $\mathrm{OB}, \mathrm{PC}$, and DQ-OVA results in PMN isolated from blood of dairy cows between 5 and $21 \mathrm{~d}$ post partum. Results of the DQ-OVA validation assay were assessed with mixed linear regression models. Pearson correlation tests and kappa values for agreement were used to associate the MFI between each PMN function assay (OB, PC, and DQ-OVA). For the validation assay (9 cows in 3 replicates), PMN incubated with DQ-OVA were stimulated with IFN- $\gamma$ or inhibited with cytochalasin $\mathrm{D}$, and fluorescence was compared with untreated PMN. Stimulated and inhibited PMN had greater (970 \pm 160 units) and lesser (593 \pm 55 units) MFI relative to untreated PMN $(791 \pm 154$ units), respectively, indicating that DQ-OVA fluorescence reflected enhanced or reduced endocytic and proteolytic function. To associate the MFI outcomes among OB, PC, and DQ-OVA, 153 samples from 40 cows were analyzed. Results showed significant, although weak association between DQ-OVA and PC MFI (Pearson r $=0.16)$. When values of MFI were categorized according to the first ("high" PMN functionality), second and

Received March 26, 2019

Accepted June 18, 2019.

*Corresponding author: obogadop@uoguelph.ca third ("moderate" PMN functionality), or fourth ("low" PMN functionality) quartiles, agreement beyond chance $(\kappa)$ was moderate: $\kappa=0.38$ for DQ-OVA and OB, $\kappa=$ 0.43 for DQ-OVA and PC, and $\kappa=0.43$ for $\mathrm{OB}$ and PC. The DQ-OVA assay may complement traditional PMN functional assays because it provides additional information regarding the combination of endocytosis and proteolytic degradation, but it is not a substitute for assessment of OB or PC.

Key words: transition period, innate immunity, immune function, DQ-ovalbumin

\section{Technical Note}

At the onset of inflammation, PMN (neutrophils) form the first line of cellular innate immune defense against invading pathogens (Paape et al., 2003). Among other functional mechanisms, PMN have the capacity to engulf pathogens through phagocytosis (PC) and degrade them through the release of reactive oxygen species from cytoplasmic granules (Lee et al., 2003). Increasing evidence suggests that PMN also have functions that contribute to adaptive immune response through interaction with antigen-presenting cells (APC), as well as the ability to act as APC themselves (Jaillon et al., 2013; Vono et al., 2017). Surface expression of antigenpresenting markers can be induced in human PMN by incubation with granulocyte-macrophage colony stimulating factor, IFN- $\gamma$, IL-3, and tumor necrosis factor- $\alpha$ (Vono et al., 2017). In dairy cows, deficits in PMN function have been linked to diseases such as retained placenta, metritis, and mastitis (Gilbert et al., 1993; Kimura et al., 2002; Sordillo, 2005). The assessment of PMN function is crucial for the evaluation of treatments aimed at overcoming immune cell dysfunction and understanding both innate and adaptive immune responses in transition cows.

Several methods for evaluating PMN in vitro function in cattle have been described, but flow cytometry has emerged as the method of choice due to its reliability and repeatability (Kampen et al., 2004). Functional 
PMN assays using flow cytometry are widely used in dairy cattle to evaluate $\mathrm{PC}$ and oxidative burst $(\mathbf{O B}$; Kampen et al., 2004; Martinez et al., 2012; Miltenburg et al., 2018). Characterization of PC involves measuring the capacity of fluorescent-labeled PMN to engulf fluorescent beads (Lehmann et al., 2000), while OB is monitored by measuring fluorescent-labeled PMN fluorescence intensity after stimulation, for example, with phorbol myristate acetate (PMA). Highly unstable oxygen metabolites released by PMN are in an electronically excited state, and excitation is accompanied by an increase in median fluorescence intensity (MFI; Mehrzad et al., 2004). However, less information is available on methods to evaluate the proteolytic degradative ability of PMN in postpartum dairy cattle.

DQ-ovalbumin (DQ-OVA; Thermo Fisher Scientific, Eugene, OR) is a self-quenching conjugate of ovalbumin that exhibits bright green fluorescence upon proteolytic degradation of boron-dipyrromethene difluoride and was originally developed for the study of antigen processing and presentation (Underhill and Goodridge, 2012). The use of DQ-OVA to evaluate pathogen handling after endocytosis could reveal an additional characteristic of PMN functioning as APC, which could provide additional information about the immune function status of dairy cows. Thus, in this study we aimed to validate the use of DQ-OVA as a potential functional assay of PMN proteolytic degradation and antigen processing. To comprehensively evaluate PMN function, we analyzed PMN with $\mathrm{OB}, \mathrm{PC}$, and DQ-OVA assays in blood from postpartum dairy cows. We hypothesized that DQ-OVA MFI would be increased in PMN activated with IFN- $\gamma$ and decreased in PMN inhibited with cytochalasin D. Because proteolytic degradation and antigen presentation occur after endocytosis, we further hypothesized that a correlation would exist between DQ-OVA and PC and between DQ-OVA and OB.

This experiment formed part of a larger study to assess the effect of anti-inflammatory treatment on the metabolic status of healthy postpartum cows. Cow handling and blood sampling were approved as per the animal utilization protocol of the University of Guelph Animal Care Committee. For the DQ-OVA validation assay, a total of 9 blood samples (1 sample per cow) from postpartum Holstein cows (from 5 to 21 DIM) were used. This sample size can detect a proportional difference of $30 \%$ between control with stimulated (Marchi et al., 2014) or inhibited (Dicker et al., 2018) PMN MFI (significance level $=0.05$, power $=0.8$ ). After validation of the DQ-OVA, 160 blood samples from 40 postpartum Holstein cows (4 samples per cow: 5, 10, 14, and $21 \mathrm{DIM}$ ) were analyzed with $\mathrm{OB}, \mathrm{PC}$, and DQ-OVA assays. This sample size is able to detect a correlation of $\mathrm{r}=0.2$ with $95 \%$ confidence and $80 \%$ power between PMN assays (Kampen et al., 2004). Samples were obtained from April to August 2018 at the University of Guelph Livestock Research and Innovation Centre Dairy Facility (Elora, ON, Canada). Cows were managed according to the guidelines set by the Dairy Farmers of Canada (Ottawa, ON, Canada) and the National Farm Animal Care Council (Lacombe, AB, Canada; DFC-NFACC, 2009).

Blood samples were collected from coccygeal vessels, using 20-gauge, 2.54-cm hypodermic needles, into sterile glass tubes containing acid citrate dextrose (Vacutainer, Becton Dickinson, Franklin Lakes, NJ). Blood tubes were gently inverted 10 times to ensure adequate mixing. Samples were placed on ice for transport and processed within $3 \mathrm{~h}$ of collection. Eight milliliters of sample with anticoagulant was diluted with $20 \mathrm{~mL}$ of $1 \times$ concentrated PBS at room temperature (18 to $21^{\circ} \mathrm{C}$ ) and gently mixed by inverting 10 times. Eight milliliters of Ficoll-Paque PLUS (General Electric Healthcare Bio-Sciences AB, Uppsala, Sweden) at room temperature were overlaid on the diluted blood sample and centrifuged at $700 \times g$ for $30 \mathrm{~min}$. The plasma and buffy coat were removed by pipetting, and erythrocytes were lysed with 6 volumes of sterile cold water using gentle inversion for $45 \mathrm{~s}$. Three volumes of $3 \times$ concentrated PBS were added to reestablish osmolarity, and the samples were then gently mixed for $45 \mathrm{~s}$ and centrifuged at $4^{\circ} \mathrm{C}$ at $500 \times g$ for $10 \mathrm{~min}$. The supernatant was removed by pipetting, and the sample was washed with $1 \times \mathrm{PBS}$ and centrifuged again at $4^{\circ} \mathrm{C}, 500 \times g$ for $10 \mathrm{~min}$ if visible hemoglobin remained in the cell pellet. Once free of visible hemoglobin, the cell pellet was resuspended in $500 \mu \mathrm{L}$ of $1 \times \mathrm{PBS}$, and a hemocytometer chamber and trypan blue exclusion were used to assess cell concentration and viability, with only samples with $>90 \%$ PMN viability being accepted. The pelleted PMN were diluted to a concentration of $1 \times 10^{6}$ in $1 \times$ PBS. Cytospin slides were prepared and stained (Wright's), and cells were tested for purity, with only samples containing $>80 \%$ PMN being accepted.

The DQ-OVA validation assay involved assessment of endocytosis and proteolytic degradation upon stimulation or inhibition of PMN. The experimental setup included 3 cows per replicate from 3 different experiments (9 cows in total). This functionality assay aimed to determine whether the DQ-OVA assay discriminates between cows with high PMN function (stimulated with IFN- $\gamma$ ) and cows with low PMN function (inhibited with cytochalasin D). Cell culture medium was prepared in advance and consisted of Dulbecco's modified Eagle's medium (Gibco/Thermo Fisher Scientific, Waltham, MA) supplemented with $10 \%$ filtered fetal bovine serum (FBS; Invitrogen, Burlington, ON, Canada). Polymor- 
phonuclear neutrophils were adjusted to a concentration of $1 \times 10^{6}$ in $120 \mu \mathrm{L}$ of cell culture medium and preincubated in the dark at $37^{\circ} \mathrm{C}$ under gentle agitation in 1.5-mL microcentrifuge tubes for $60 \mathrm{~min}$ (Baquero and Plattner, 2017). The preincubation groups consisted of (1) stimulation; PMN supplemented with $80 \mu \mathrm{L}(1,000$ $\mathrm{ng} / \mathrm{mL}$ ) of IFN- $\gamma$ (R\&D Systems, Minneapolis, MN), (2) inhibition; PMN supplemented with $10 \mu \mathrm{L}(10 \mu \mathrm{g} /$ $\mathrm{mL}$ ) of cytochalasin D (Sigma-Aldrich, St. Louis, MO), and (3) control; PMN without supplementation. After preincubation, cells were washed with $1 \times$ PBS and diluted with $120 \mu \mathrm{L}$ of fresh cell culture medium plus $10 \mu \mathrm{L}$ of DQ-OVA $(10 \mu \mathrm{g} / \mathrm{mL})$. An additional control consisting of freshly isolated PMN suspended in $120 \mu \mathrm{L}$ of cell culture medium at a concentration of $1 \times 10^{6}$ cells with $10 \mu \mathrm{L}$ of DQ-OVA $(10 \mu \mathrm{g} / \mathrm{mL})$ was also prepared (no preincubation group). Last, negative controls consisted of PMN $\left(1 \times 10^{6}\right)$ suspended in $120 \mu \mathrm{L}$ of cell culture medium with no DQ-OVA added. All 5 groups of cells were incubated in the dark at $37^{\circ} \mathrm{C}$ under gentle agitation in 1.5-mL microcentrifuge tubes for $45 \mathrm{~min}$ (Baquero and Plattner, 2017). After incubation, cells were washed with $1 \times$ PBS, diluted in $200 \mu \mathrm{L}$ of cold 1 $\times$ PBS, allocated to flow cytometry tubes on ice, and protected from light until analysis.

For the OB and PC assays, samples were prepared as described by Miltenburg et al. (2018). For the OB assays, $200 \mu \mathrm{L}$ of $1 \times$ PBS with $10 \%$ FBS was added to the control tube, and $200 \mu \mathrm{L}$ of PMA (Sigma-Aldrich) diluted in PBS/FBS for a total of $25 \mathrm{ng} / \mathrm{mL}$ of PMA was added to stimulate $\mathrm{OB}$ in the treatment tube. For PC assays, $200 \mu \mathrm{L}$ of reconstituted PMN $\left(1 \times 10^{6}\right)$ was incubated with fluorescently labeled $1-\mu \mathrm{m}$ beads (TransFluo-Spheres Fluorescent Microspheres, Molecular Probes; Thermo Fisher Scientific, Eugene, OR) and $50 \mu \mathrm{L}$ of activated normal cow serum for $30 \mathrm{~min}$ at $37^{\circ} \mathrm{C}$ in the dark. Each sample had a negative control of PMN incubated without fluorescent beads. For the activated normal cow serum preparation, $100 \mathrm{mg}$ of zymosan A from Saccharomyces cerevisiae (Sigma-Aldrich, Winston Park, Oakville, ON, Canada) was added per $10 \mathrm{~mL}$ of pooled blood serum from healthy cows, incubated at $37^{\circ} \mathrm{C}$ for 60 min under gentle rotation, and centrifuged at $2,500 \times g$ for $15 \mathrm{~min}$. For the DQ-OVA assay, $120 \mu \mathrm{L}$ of cell culture medium containing $1 \times 10^{6}$ PMN served as the negative control and test samples were supplemented with $10 \mu \mathrm{L}$ of DQ-OVA $(10 \mu \mathrm{g} /$ $\mathrm{mL})$.

All samples were analyzed by flow cytometry (FACSCanto, BD Biosciences, San Jose, CA). The threshold was set at 10,000 events in a gate around the population of interest (PMN) on cytograms of forward versus side scatter. Oxidative burst was measured at $530 \mathrm{~nm}$ and PC at $780 \mathrm{~nm}$ on a log scale (Supplemental Figure
S1, panels C and D; https://doi.org/10.3168/jds.2019 -16687). The bright green fluorescence exhibited by the ovalbumin labeled with the $\mathrm{pH}$-insensitive fluorescent dye, boron-dipyrromethene difluoride, was measured at $530 \mathrm{~nm}$ on a log scale (Supplemental Figure S1, panel B). Data were analyzed using FlowJo software (Tree Star, Ashland, OR). A gate was drawn around the PMN population (Supplemental Figure S1, panel A) and cytograms were transformed to histograms. Given that PMN neutrophils represent $>90 \%$ of the granulocyte population in the blood of cows, we inferred that this gating strategy referred primarily to PMN neutrophils (Martinez et al., 2012). Control and treatment PMN histograms were visualized to corroborate the functionality of the assays. The difference in the MFI of PMN that underwent $\mathrm{OB}, \mathrm{PC}$, or proteolytic degradation (DQ-OVA) relative to the MFI of the respective negative control (autofluorescence) was calculated.

Data were exported to $\mathrm{R}$ version 3.3.0 (R Core Team, Vienna, Austria), which was used for all statistical analyses. The distribution of continuous outcomes was evaluated using the Shapiro-Wilk test. To assess differences between PMN functionality in the DQ-OVA validation, the MFI of each group (IFN- $\gamma$, cytochalasin $\mathrm{D}$, preincubation control, no preincubation control, and non-DQ-OVA negative control) were fitted in mixed linear regression models (function lmer, package lme4) using the replicate as the random effect. Pearson correlation tests (function cor, package Hmisc) were used to associate the MFI outcome between each PMN function assay (OB, PC, and DQ-OVA). The MFI outcomes of the DQ-OVA PMN test were also divided into 3 groups: (1) first quartile ("high" PMN functionality), (2) second and third quartile ("moderate" PMN functionality), and (3) fourth quartile ("low" PMN functionality). At each level, Pearson correlation tests were used to associate the MFI outcome of DQ-OVA with the corresponding level (high, moderate, and low) of $\mathrm{OB}$ or $\mathrm{PC}$ assays. Each functional level was categorized and compared with each other to determine interassay Cohen's kappa agreement ( $\kappa$; function confusionMatrix, package caret). Continuous values were expressed as least squares means \pm standard errors. The level of significance was set at $P \leq 0.05$.

For the DQ-OVA validation assay (3 cows per replicate in 3 different experiments), stimulated PMN (preincubated with IFN- $\gamma$ ) had higher MFI than nonstimulated PMN and inhibited PMN (preincubated with cytochalasin D) groups, and inhibited PMN had lower MFI than nonstimulated PMN groups (Figure 1 ). From the 160 blood samples acquired to compare the different PMN functions, 7 were discarded due to spontaneous PMN activation (in vitro cell aggregation). The correlation (Pearson) graphs in Figure 2 depict 


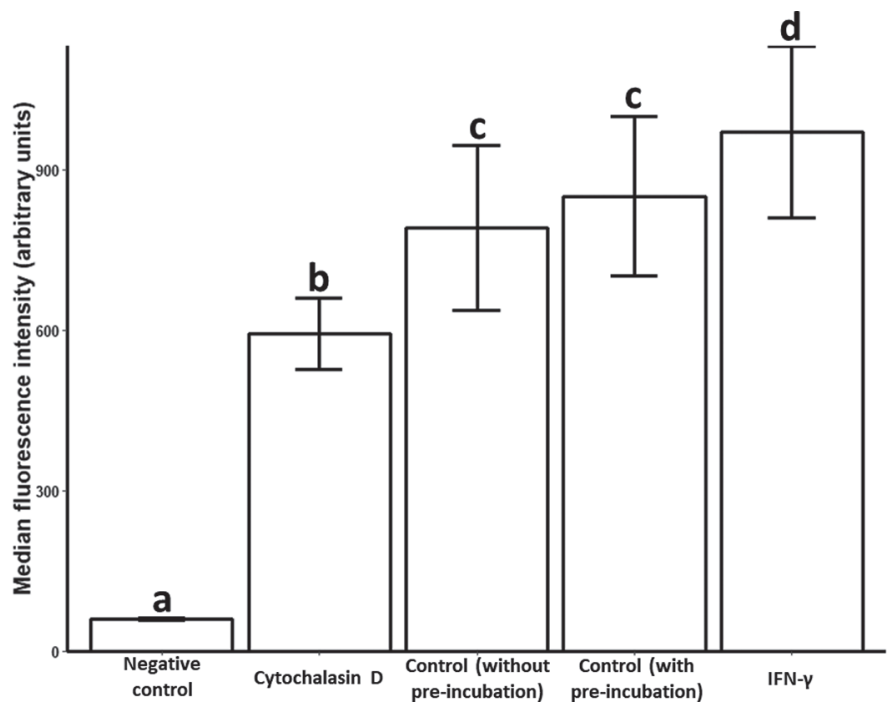

Figure 1. Least squares means \pm standard error of the median fluorescence intensity of PMN (neutrophils) cocultured with DQovalbumin (DQ-OVA) at $37^{\circ} \mathrm{C}$ for 45 min after preincubation with IFN- $\gamma$ (stimulated PMN), preincubation with cytochalasin D (inhibited PMN), preincubation without stimulant or inhibitor, and DQ-OVA without prior incubation. Negative control consisted of PMN incubated without DQ-OVA (autofluorescence). Different letters (a-d) represent differences $(P<0.05)$ between groups. Total number of cows, $\mathrm{n}$ $=9$ (3 cows per replicate in 3 different experiments).

the relationships within each assay. A weak positive correlation existed between DQ-OVA and PC results ( $\mathrm{r}$ $=0.16, P=0.04$; Figure $2 \mathrm{~B}$ ), and between DQ-OVA and PC MFI $(\mathrm{r}=0.23, P=0.05)$ at the high PMN function level. No significant correlations $(\mathrm{r}=-0.13$ to 0.1 ) were found between the other assays at any functionality category (high, moderate, or low; $P>0.3$ ). The $\kappa$ values for agreement between high, moderate, and low PMN function were $\kappa=0.38$ for DQ-OVA and $\mathrm{OB}, \kappa=0.43$ for DQ-OVA and PC, and $\kappa=0.43$ for $\mathrm{OB}$ and $\mathrm{PC}$.

Proteolytic degradation is a crucial step for antigen processing in the immune response, and it may be overlooked when assessing only $\mathrm{OB}$ and PC functions of PMN. This study is the first to validate measurement of proteolytic processing of an endocytosed protein in $\mathrm{PMN}$ and to compare the findings to $\mathrm{OB}$ and $\mathrm{PC}$ results in blood-isolated PMN of postpartum dairy cows.

Based on the results from flow cytometry, exposure of PMN to IFN- $\gamma$ conserved their morphology (visualized in the forward and side scatter gating for the PMN) and increased their endocytic and proteolytic activity during subsequent incubation with DQ-OVA. Interferon- $\gamma$, which is one of the most potent pleiotropic cytokines, upregulates PMN endocytosis, PC, or both by an alternative pathway to PMA (Lieser et al., 1995; Marchi et al., 2014). Preincubation with cytochalasin D reduced the endocytic activity of PMN (Brinkmann et al., 2004; Dicker et al., 2018), which was reflected by decreased MFI of DQ-OVA. The use of DQ-OVA to assess PMN function in postpartum dairy cows is a novel approach to evaluate the endocytic and proteolytic capacities of PMN. Because ovalbumin is internalized via the mannose receptor-mediated endocytosis
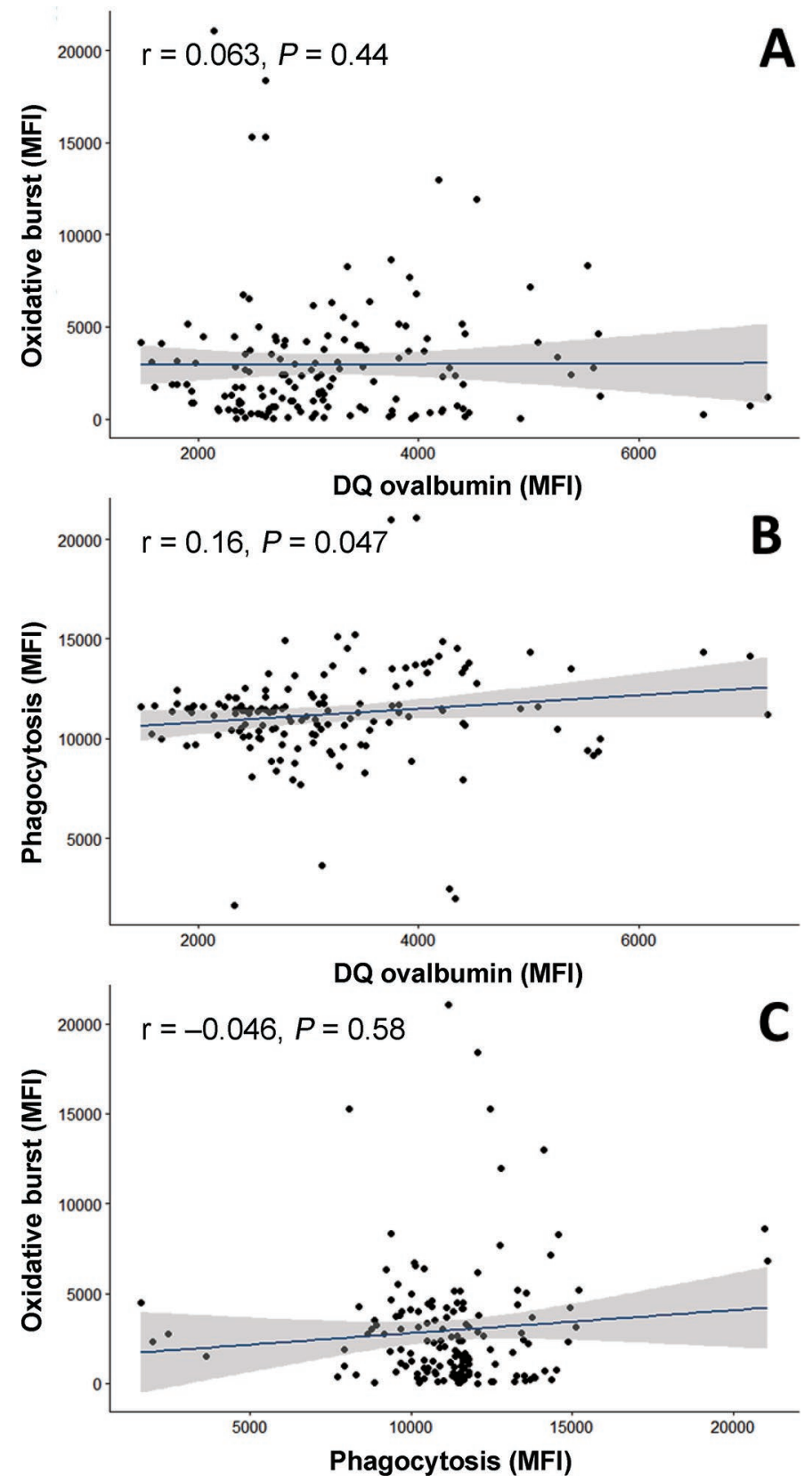

Figure 2. Correlation graphs illustrating the relationship of PMN (neutrophil) leukocyte median fluorescence intensity (MFI) between (A) DQ-ovalbumin (DQ-OVA) and oxidative burst, (B) DQ-OVA and phagocytosis, and (C) phagocytosis and oxidative burst. The blue lines and the gray shadings are the regression and the $95 \%$ confidence interval between assays, respectively $(\mathrm{n}=153$ blood samples for each graph). 
pathway (specific for APC cells; Janicka et al., 1994), future studies should explore the potential role of PMN as APC.

As indicated by their significant positive correlation, DQ-OVA and PC assays measured the common engulfing capacity of PMN, but the relationship was weak. This weak association is probably due to the nature of the PMN function assays because PC of fluorescent beads does not reflect proteolysis of engulfed material. On the other hand, DQ-OVA emits fluorescence only after proteolytic degradation of boron-dipyrromethene difluoride, a pH-insensitive fluorescent dye bound to ovalbumin (i.e., after both endocytosis and degradation). The difference between assays may also be associated with the cellular process used to internalize fluorescent beads and ovalbumin. Although the PC assay reflects the capacity of PMN to phagocyte insoluble beads, DQ-OVA is a soluble protein that is acquired by endocytosis (or pinocytosis) and rapidly degraded by PMN organelles. Our results showed no correlation between $\mathrm{OB}$ and $\mathrm{PC}$ or between OB and DQ-OVA assays. This outcome is not surprising because PC and DQ-OVA measure the PMN engulfment capacity, while OB evaluates the release of reactive oxygen species. This dissimilarity between PMN assays is in accordance with other findings, in which a treatment effect was identified in only 1 of 2 PMN functions (Miltenburg et al., 2018; Martinez et al., 2018). Therefore, our findings suggest that each assay measures different aspects of PMN function, and that these functions are not necessarily concordant in PMN from dairy cows in the transition period.

In summary, the DQ-OVA assay is suitable for testing the combined endocytic and proteolytic functions of PMN in dairy cows. We found a weak correlation between DQ-OVA and PC results and no association between $\mathrm{OB}$ and DQ-OVA or between $\mathrm{OB}$ and PC assays. Hence, we recommend performing more than one assay when evaluating PMN function in dairy cows. The DQ-OVA assay may complement traditional PMN functional assays because it provides additional information regarding the combination of endocytosis and proteolytic degradation, but it is not a substitute for assessment of OB or PC. Further research should be directed at better understanding how PMN may function as APC and contribute to specific immune responses in the postpartum dairy cow.

\section{REFERENCES}

Baquero, M. M., and B. L. Plattner. 2017. Bovine $\mathrm{WC}^{+}$and $\mathrm{WC} 1^{\text {neg }}$ $\gamma \delta \mathrm{T}$ lymphocytes influence monocyte differentiation and monocyte-derived dendritic cell maturation during in vitro Mycobacterium avium subspecies paratuberculosis infection. Front. Immunol. $8: 534$.
Brinkmann, V., U. Reichard, C. Goosmann, B. Fauler, Y. Uhlemann, D. S. Weiss, Y. Weinrauch, and A. Zychlinsky. 2004. Neutrophil extracellular traps kill bacteria. Science 303:1532-1535.

DFC-NFACC. 2009. DFC-NFACC (Dairy Farmers of Canada and the National Farm Animal Care Council) Code of Practices for the Care and Handling of Dairy Cattle. Dairy Farmers of Canada, Ottawa, Ontario, Canada.

Dicker, A. J., M. L. Crichton, E. G. Pumphrey, A. J. Cassidy, G. Suarez-Cuartin, O. Sibila, E. Furrie, C. J. Fong, W. Ibrahim, G. Brady, G. G. Einarsson, J. S. Elborn, S. Schembri, S. E. Marshall, C. N. A. Palmer, and J. D. Chalmers. 2018. Neutrophil extracellular traps are associated with disease severity and microbiota diversity in patients with chronic obstructive pulmonary disease. J. Allergy Clin. Immunol. 141:117-127.

Gilbert, R. O., Y. T. Gröhn, P. M. Miller, and D. J. Hoffman. 1993. Effect of parity on periparturient neutrophil function in dairy cows. Vet. Immunol. Immunopathol. 36:75-82.

Jaillon, S., M. R. Galdiero, D. Del Prete, M. A. Cassatella, C. Garlanda, and A. Mantovani. 2013. Neutrophils in innate and adaptive immunity. Semin. Immunopathol. 35:377-394.

Janicka, M., P. A. Chindemi, W. L. Hu, and E. Regoeczi. 1994. Effect of transferrin on the degradation of glycoproteins bearing a hybrid or high-mannose glycan by alveolar macrophages. Exp. Cell Res. $215: 17-22$.

Kampen, A. H., T. Tollersrud, S. Larsen, J. A. Roth, D. E. Frank, and A. Lund. 2004. Repeatability of flow cytometric and classical measurement of phagocytosis and respiratory burst in bovine polymorphonuclear leukocytes. Vet. Immunol. Immunopathol. 97:105-114.

Kimura, K., J. P. Goff, M. E. Kehrli, and T. A. Reinhardt. 2002. Decreased neutrophil function as a cause of retained placenta in dairy cattle. J. Dairy Sci. 85:544-550.

Lee, W. L., R. E. Harrison, and S. Grinstein. 2003. Phagocytosis by neutrophils. Microbes Infect. 5:1299-1306.

Lehmann, A. K., S. Sørnes, and A. Halstensen. 2000. Phagocytosis: Measurement by flow cytometry. J. Immunol. Methods 243:229242.

Lieser, M. J., R. A. Kozol, and S. D. Tennenberg. 1995. Interferongamma primes neutrophil-mediated gastric surface cell cytotoxicity. Am. J. Physiol. 268(5 Pt. 1):G843-G848.

Marchi, L. F., R. Sesti-Costa, M. D. Ignacchiti, S. Chedraoui-Silva, and B. Mantovani. 2014. In vitro activation of mouse neutrophils by recombinant human interferon-gamma: Increased phagocytosis and release of reactive oxygen species and pro-inflammatory cytokines. Int. Immunopharmacol. 18:228-235.

Martinez, N., C. A. Risco, F. S. Lima, R. S. Bisinotto, L. F. Greco, E. S. Ribeiro, F. Maunsell, K. Galvão, and J. E. P. Santos. 2012. Evaluation of peripartal calcium status, energetic profile, and neutrophil function in dairy cows at low or high risk of developing uterine disease. J. Dairy Sci. 95:7158-7172.

Martinez, N., R. Rodney, E. Block, L. L. Fernandez, C. D. Nelson, I. J. Lean, and J. E. P. Santos. 2018. Effects of prepartum dietary cation-anion difference and source of vitamin D in dairy cows: Health and reproductive responses. J. Dairy Sci. 101:2563-2578.

Mehrzad, J., H. Dosogne, E. Meyer, R. Heyneman, and C. Burvenich. 2001. Respiratory burst activity of blood and milk neutrophils in dairy cows during different stages of lactation. J. Dairy Res 68:399-415.

Miltenburg, C. L., T. F. Duffield, D. Bienzle, E. Scholtz, and S. J. LeBlanc. 2018. The effect of prepartum feeding and lying space on metabolic health and immune function. J. Dairy Sci. 101:52945306 .

Paape, M. J., D. D. Bannerman, X. Zhao, and J. W. Lee. 2003. The bovine neutrophil: Structure and function in blood and milk. Vet. Res. 34:597-627.

Sordillo, L. M. 2005. Factors affecting mammary gland immunity and mastitis susceptibility. Livest. Prod. Sci. 98:89-99.

Underhill, D. M., and H. S. Goodridge. 2012. Information processing during phagocytosis. Nat. Rev. Immunol. 12:492-502.

Vono, M., A. Lin, A. Norrby-Teglund, R. A. Koup, F. Liang, K. Loré, and L. Loré. 2017. Neutrophils acquire the capacity for antigen presentation to memory CD4 $1 \mathrm{~T}$ cells in vitro and ex vivo. Blood 129:1991-2001. 